\title{
Using DEVS and CELL-DEVS for Modelling of Information Impact on Individuals in Social Network
}

\author{
Youssef Bouanan, Merouane Bouhamidi El Alaoui, Gregory Zacharewicz, \\ and Bruno Vallespir \\ University of Bordeaux, Lab. IMS, UMR 5218, 33405 Talence, France \\ \{youssef.bouanan, gregory.zacharewicz, \\ Bruno.vallespir\}@ims-bordeaux.fr, alaoui.merouane@gmail.com
}

\begin{abstract}
We observe that major works about modelling and simulation within social science, especially for social, organizational and cultural influences on opinion information spreading over a population, do not use specification languages to describe their models. These models are specified in the shape of math formulas and then directly coded using classical programming languages. The specification language can be a missing link. For instance, the DEVS formalism (Discrete EVent system Specifications) being general enough to represent dynamical systems, can provide an operational semantics applicable to this domain. These models independent from implementation are easily reusable. In this article, we recall first the use of discrete modelling approaches in the social influence. Then we present models for human information treatment and propagation using DEVS and Cell-DEVS (Cellular DEVS). Finally, we present a simulation transposed from epidemic models to the impact of information on individuals using CD++, a simulation tool for DEVS and Cell-DEVS.
\end{abstract}

Keywords: DEVS Formalism, Information Impact, CELL-DEVS, Human Behaviour Modelling.

\section{$1 \quad$ Introduction and Motivation}

The reactions of populations to the dissemination and propagation of information are, up to now, not modeled appropriately. There is however an interest in the ability to simulate and accurately measure the impact of information on population. The SICOMORES project objective is to provide solutions to artificially generate structured social networks of realistic population and simulate the effects of information on population, with a propagation algorithm of the effects across networks. The intention is to go further than current models which generally reduce the individuals of a population as simple obstacles or information transmitters without enough nuances in their behaviour and the influence they can have on a message. This paper intends to present the premise of modelling and simulation of the impact of influence activities on individuals in social networks using Cell DEVS M\&S formalism.

Cell-DEVS is a combination of CA (Cellular Automata) with the DEVS (Discrete EVent system Specifications) formalism that allows the definition of complex cell

B. Grabot et al. (Eds.): APMS 2014, Part III, IFIP AICT 440, pp. 409-416, 2014.

(C) IFIP International Federation for Information Processing 2014 
based systems. It appears especially suited to this study that takes into account several layers of social graph related with geographical networks. CD++ is a modelling and simulation tool that implements DEVS and Cell-DEVS. We use CD++ to build a model of influence of information on individuals in social networks. In more detail, this paper will participate in the definition of a set of models that addresses the entities and the structure of a population. It will present a state of the art of modelling and simulation approaches to describe a social system at multiple levels including multiagent systems (MAS) and discrete modelling approaches. In addition, it will provide models of individuals and groups of individuals characterized by a set of state variables (e.g. Using Maslow to construct the behaviour of an individual) and the mesh between the individuals within a social network.

\section{Background}

\subsection{DEVS Formalism}

The DEVS formalism for modelling and simulation (Zeigler et al., 2000) is based on discrete events, and provides a framework with mathematical concepts based on the sets theory and systems theory concepts to describe the structure and the behaviour of a system. With DEVS, there is an explicit separation between a model and its simulator: once a model is defined, it is used to build a simulator (i.e. a device able to execute the model's instructions). DEVS knows two kinds of models: the atomic models, which describe behaviour, and the coupled models which describe a hierarchy.

The tiniest element in DEVS formalism is the atomic model. It is specified as:

$$
\mathrm{AM}=<\mathrm{X}, \mathrm{Y}, \mathrm{S}, \mathrm{ta}, \delta \text { int }, \delta \text { ext, } \lambda>
$$

The semantics for this definition is given as follows. At any time, a DEVS atomic model is in a state $s \in \mathrm{S}$. In the absence of external events, the model will stay in this state for the duration specified by $\mathrm{ta}(\mathrm{s})$. When the elapsed time $\mathrm{e}=\mathrm{ta}(\mathrm{s})$, the state duration expires and the atomic model will sent the output $\lambda(\mathrm{s})$ and performs an internal transition to a new state specified by $\delta$ int (s). Transitions that occur due to the expiration of $\mathrm{ta}(\mathrm{s})$ are called internal transitions.

However, state transition can also happen due to arrival of an external event which will place the model into a new state specified by $\delta$ ext $(\mathrm{s}, \mathrm{e}, \mathrm{x})$; where $\mathrm{s}$ is the current state, $\mathrm{e}$ is the elapsed time, and $\mathrm{X}$ is the input value. The time advance function ta(s) can take any real value from 0 to $\infty$. A state with ta(s) value of zero is called transient state, and on the other hand, if $\operatorname{ta}(s)$ is equal to $\infty$ the state is said to be passive, in which the system will remain in this state until receiving an external event.

Table 1 show the graphical notation to define the behaviour of atomic models.

Table 1. DEVS graphical notation

\begin{tabular}{|c|c|c|}
\hline State & Internal Transition & External Transition \\
\hline $\left.\begin{array}{c}\mathrm{Name} \\
\mathrm{Ta}\end{array}\right)$ & $\left(\begin{array}{c}\mathrm{q} 0 \\
\mathrm{Ta}=\mathrm{e} 0\end{array}\right)$ & $\mathrm{q0!20}$ \\
\hline
\end{tabular}




\subsection{CELL-DEVS}

Cell-DEVS (Wainer, 2009) has extended the DEVS formalism, allowing the implementation of cellular models with timing delays. It improves execution performance of cellular models by using a discrete-event approach. It also enhances the cell's timing definition by making it more expressive. Each cell is defined as an atomic model using timing delays, and it can be later integrated to a coupled model representing a cell space, as showed in Figure 1.

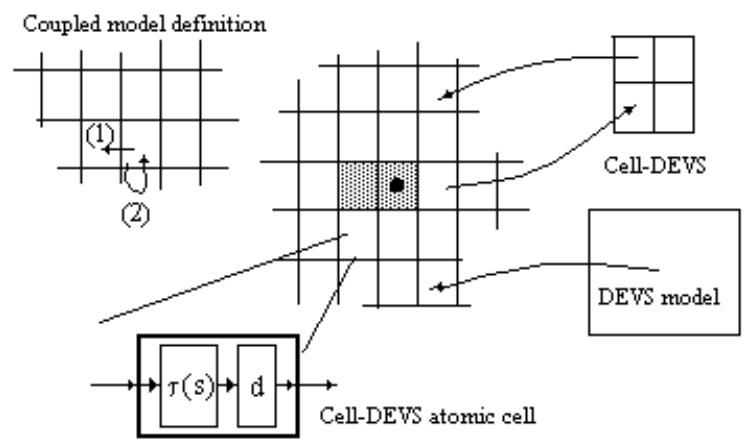

Fig. 1. Informal Description of Cell-DEVS

Once the cell behaviour is defined, a coupled Cell-DEVS can be created by putting together a number of cells interconnected with their neighbors. A cellular model is a lattice of cells holding state variables and a computing apparatus, which is in charge of updating the cell state according to a local rule. This is done using the present cell state and those of a finite set of nearby cells (called its neighborhood).

Each cell uses $\mathrm{N}$ inputs to compute its next state. These inputs, which are received through the model's interface, activate a local computing function (t). A delay (d) can be associated with each cell. The state (s) changes can be transmitted to other models, but only after the consumption of this delay. Two kinds of delays can be defined: transport delays model a variable commuting time (every state change is transmitted), and inertial delays, which have preemptive semantics (scheduled events can be discarded).

\section{$2.3 \quad$ CD++ Toolkit}

CD++ (Wainer, 2002) is a modelling and simulation toolkit that implements DEVS and Cell-DEVS theory. Atomic models can be defined using a state-based approach (coded in C++ or an interpreted graphical notation), while coupled and Cell-DEVS models are defined using a built-in specification language. We will show the basic features of the tool through an example of application. $\mathrm{CD}++$ also includes an interpreter for Cell-DEVS models. The model specification includes the definition of the size and dimension of the cell space, the shape of the neighborhood and borders. The cell's local computing function is defined using a set of rules with the form: 
\{POSTCONDITION\} \{DELAY\} \{PRECONDITION\}. These indicate that when the PRECONDITION is satisfied, the state of the cell will change to the designated POSTCONDITION, whose computed value will be transmitted to other components after consuming the DELAY.

\section{Human Behaviour Modelling}

\subsection{General Approach}

Human Behaviour Modelling is an important area of computational science with implications not only for social sciences, but also for economics, epidemiology and other fields. Scientific literature abounds in heterogeneous and highly specialized, theoretically founded concepts of human cognition, emotion and other behaviour aspects. There are many lines of research on such models, which span several disciplines, have different goals, and often use different terminologies and various approaches. Human behaviour modelling or human behaviour representation (HBR) is an important field of study in military service research (Fei et al., 2007), robotics [(Kubota and Nichida, 2006)], brain-computer interface (BCI), human machine interface (HMI) and some specially oriented anthropology studies. Human behaviour models are often represented by finite state machines, rules, fuzzy rules (Dorsey and Coovert, 2003), artificial neural networks, multi-agent based modelling (Sun, 2007). The need for a variety of modelling paradigms stems from the fact that the different domains of knowledge needed to represent human behaviour cannot be done by only one paradigm.

\subsection{Using DEVS for Human Behaviour Modelling}

Human behaviour can be difficult to understand and predict, thus it can be qualified as a complex system. DEVS is a well-defined formalism which has numerous advantages over other formalisms in the modelling of complex dynamic systems. A few related works have provided DEVS models of human behaviour that we will use with slight modifications; (Seck, 2004) present a DEVS based framework for the modelling and simulation of human behaviour with the influence of stress and fatigue, (Faucher, 2012) proposed a first approach using G-DEVS formalism for CivilMilitary Cooperation actions (CIMIC) and Psychological actions (PSYOPS), which are actions of influence that take precedence over combat. The purpose of this work is to go beyond previous works by providing a simple model but more performant and accurate which will allow us not only to model the behaviour of an individual, but also the simulation of the propagation of an information among a group of individuals and its influence on their behaviour.

This model (Figure 2) is describing the influence of message on the behaviour of an individual and potentially its dissemination. The first state consists in being in 
contact with another agent in its social network neighbourhood and calculating the strength of connexion between them. When the message is received, it creates an impact on the individual which change its behaviour eventually depending on the strength of the message (Faucher, 2012). Then, if the message strength is still strong enough the receiver is preparing on its turn to transmit the message to its network neighbours considered as target info.

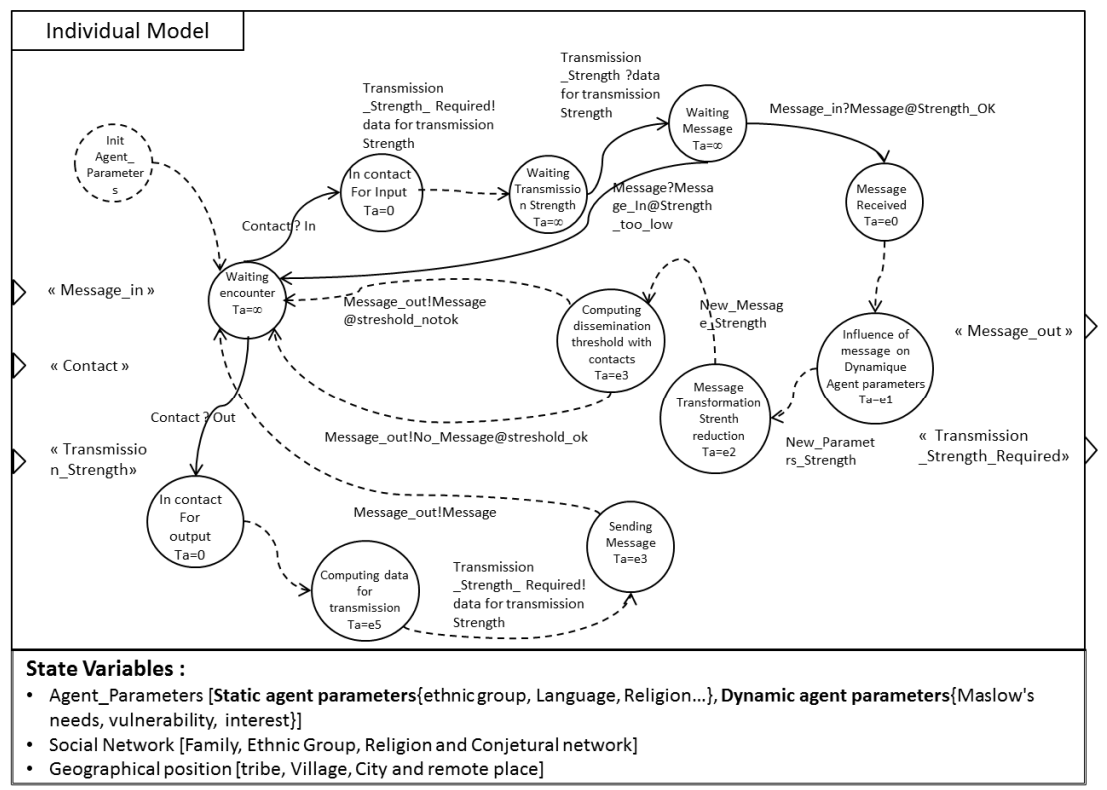

Fig. 2. DEVS individual model

\section{$4 \quad$ Using CELL-DEVS to Simulate Information Propagation}

\subsection{From Epidemics Spreading to Information Diffusion}

The phenomena of information propagation have known a great interest of researchers and still an active field of research as well (Bakshy et al., 2011). In the past, the propagation was mainly studied in the epidemiological field to better understand the process of propagation of infection in certain conditions. Therefore, the majority of recent works related to information diffusion are based on the epidemic researches. There is a basic analogy (Table 2) between the dissemination of information among a group of individuals and the transmission of infectious disease between the individuals themselves. In the two cases, something is communicated through a given contact. 
Table 2. Analytical model parameters in epidemiology versus diffusion

\begin{tabular}{|c|c|c|}
\hline & Epidemiology & Information diffusion \\
\hline $\mathrm{N}$ & Number of individuals & Number of individuals \\
\hline $\mathrm{S}(\mathrm{t})$ & $\begin{array}{l}\text { Number of susceptible individuals at } \\
\text { time } t\end{array}$ & $\begin{array}{l}\text { Number of susceptible individuals at } \\
\text { time } t\end{array}$ \\
\hline $\mathrm{I}(\mathrm{t})$ & $\begin{array}{l}\text { Number of infectious individuals at } \\
\text { time } t\end{array}$ & $\begin{array}{l}\text { Number of individuals at time } t \text { that } \\
\text { carry the information }\end{array}$ \\
\hline $\mathrm{X}$ & $\begin{array}{l}\text { Contacts per unit of time and per indi- } \\
\text { vidual }\end{array}$ & $\begin{array}{l}\text { Contacts per unit of time and per } \\
\text { individual }\end{array}$ \\
\hline$\beta$ & $\begin{array}{l}\text { the probability of transmission in a } \\
\text { contact between an infective and a } \\
\text { susceptible }\end{array}$ & $\begin{array}{l}\text { the probability of transmission in a } \\
\text { contact between an information holder } \\
\text { and a susceptible }\end{array}$ \\
\hline$a=\beta x / N$ & Infection rate & Infection rate \\
\hline $\begin{array}{l}\alpha= \\
\mathrm{a}^{*} \mathrm{I}(\mathrm{t})\end{array}$ & $\begin{array}{l}\text { Force of infection: The probability per } \\
\text { unit of time for a susceptible to become } \\
\text { infective }\end{array}$ & $\begin{array}{l}\text { Transmission Strength: The probabili- } \\
\text { ty per unit of time for a node to re- } \\
\text { ceive the information }\end{array}$ \\
\hline
\end{tabular}

\subsection{CELL-DEVS Implementation}

The following figure shows the procedure steps of model executions in CD++. We used and adapted according to the transposition proposed in the Table 2 an existing model of virus spreading defined with CELL-DEVS (Shang, 2005), (CELL-DEVS, 2014).
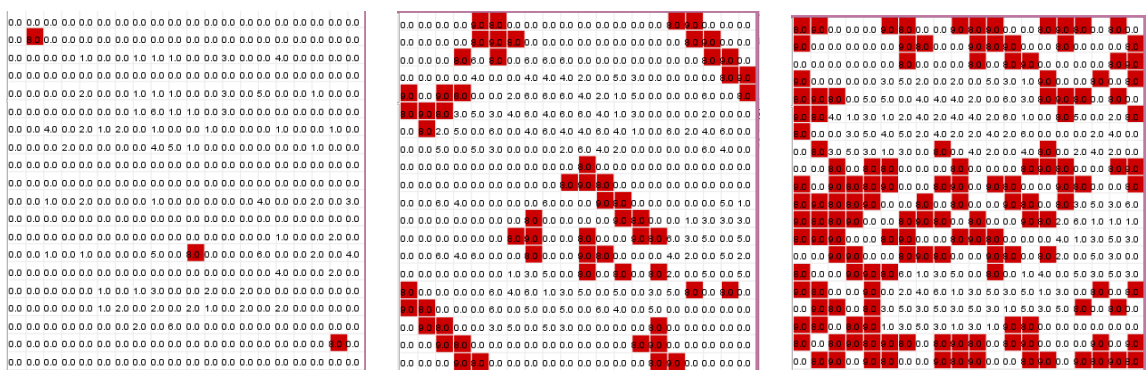

Fig. 3. Execution result at time 00:00:00:000; 00:00:00:500; 00:00:01:000

The model is intended to study the propagation of information within a group of individuals. Assume there is $20 \times 20$ mesh of cellular automata (CA). Each individual, residing in $(\mathrm{i}, \mathrm{j})$ node, is equipped with a number ranged from 0 to 9 indicating 
different Parameters of the individual. Each cell may represent one of two categories: one is person who did not receive the information represented by white color; the other is the person who received the message represented by red color. The individual can be opposed to the information and/or person that is transmitting the message and/or favourable to the emitter and message content. The simulation results show on the right picture of Figure 3 that depending on the opinion of individuals and the configuration of the social networks some individual can be reached by the information where some other not. This test is very simple; it is based on the abstract geographical situation of individuals and it takes into account only one dimension of a social network. We have not introduced yet the graph representation for defining the multilevel social network links between individuals or groups. Nevertheless the approach already validates the possibility to reuse epidemic algorithm for information spreading.

\section{Conclusion}

This paper introduced Formal Modelling and Simulation of the impact of Information on Individuals in Social Networks. We have presented how DEVS and Cell-DEVS can be very useful techniques for modelling and simulating of Social influence. At the individual level, the DEVS model proposed is very simple keeping raw Maslow parameters as state variables and being simply influenced by arriving messages. In its turn the individual model can transmit the message after having potentially transformed its strength according to several criteria. The Cell DEVS structure then use these models of human extremely simplified and start building on top of its connected modules to form Cell-DEVS network. In addition, the separation between the model and simulator followed by DEVS and $\mathrm{CD}++$, has enabled the modeller to concentrate on building the behavioural model on one side and preparing the spreading logic on the other side using the $\mathrm{CD}++$ toolkit. The model shows a correct, even very simple, human behaviour impact regarding information perception and treatment. Then the information spreading reusing the epidemic approaches seems accurate after some adaptations to propagate the information over the network.

\section{Perspectives}

The main perspective remains the definition of a multi-level social network. In detail the real social network is complex, it can refer for e.g. to the family environment, work, religion, geography to transport the information from one individual to another. The final result will be the combination of several networks graphs. The next step will also consider other experimental terrains, for instance enterprise and organization. 


\section{References}

1. Bakshy, E., Hofman, J.M., Mason, W.A., Watts, D.J.: Everyone's an influencer: quantifying influence on twitter. In: Proceedings of the Fourth ACM International Conference on Web Search and Data Mining, pp. 65-74 (2011)

2. CELL-DEVS Models, http: / / www. sce.carleton.ca/faculty/wainer/wbgraf / doku.php?id =model_samples: start (accessed April 2014)

3. Diekmann, O., Heesterbeek, J.A.P.: Mathematical Epidemiology of Infectious Diseases. Wiley Ser. Mathematical and Computational Biology, England (2000)

4. Ding, W., Pei, L., Li, H., Xi, N., Wang, Y.: The Effects of Time Delay of Internet on Characteristics of Human Behaviors. In: Proceedings of the 2009 IEEE International Conference on Networking, Sensing and Control, Okayama, Japan (2009)

5. Dorsey, D.W., Coovert, M.D.: Mathematical Modeling of Decision making: A Soft and Fuzzy Approach to Capturing Hard Decisions. Human Factors 45(1), 117-135 (2003)

6. Faucher, C., Zacharewicz, G., Hamri, E.M., Frydman, C.: PSYOPS and CIMIC operations: from concepts to G-DEVS models. SpringSim (TMS) 42 (2012)

7. Fei, L., Ming, Y., Guobing, S.: Verification of Human Decision Models in Military Simulations. In: Proceedings of the IEEE 2007, The First Asia International Conference on Modeling \& Simulation, pp. 363-368 (2007)

8. Kubota, N., Nishida, K.: Prediction of Human Behavior Patterns based on Spiking Neurons. In: The 15th IEEE International Symposium on Robot and Human Interactive Communication, ROMAN 2006 (2006)

9. Seck, M., Frydman, C., Giambiasi, N.: Using DEVS for Modeling and Simulation of Human Behaviour. In: Kim, T.G. (ed.) AIS 2004. LNCS (LNAI), vol. 3397, pp. 692-698. Springer, Heidelberg (2005)

10. Shang, H., Wainer, G.: A Model of Virus Spreading Using Cell-DEVS. In: Sunderam, V.S., van Albada, G.D., Sloot, P.M.A., Dongarra, J.J. (eds.) ICCS 2005. LNCS, vol. 3515, pp. 373-377. Springer, Heidelberg (2005)

11. Sun, Z.: Multi-Agent Based Modeling: Methods and Techniques for Investigating Human Behaviors. In: Proceedings of IEEE International Conference on Mechatronics and Automation (2007)

12. Wainer, G.: "CD++: A toolkit to develop DEVS models", Software - Practice and Experience, pp. 1261-1306 (2002)

13. Wainer, G.: Discrete-Event Modeling and Simulation: A Practitioner's approach. CRC Press (2009)

14. Zacharewicz, G., Frydman, C., Giambiasi, N.: G-DEVS/HLA Environment for Distributed Simulations of Workflows 84(5), 197-213 (2008)

15. Zeigler, B.P., Praehofer, H., Kim, T.G.: Theory of Modelling and Simulation. Editions Academic Press, San Diego (2000) 